\title{
310.
}

\section{NOTE ON MR JERRARD'S RESEARCHES ON THE EQUATION OF THE FIFTH ORDER.}

[From the Philosophical Magazine, vol. xxi. (1861), pp. 210-214.]

Functions of the same set of quantities which are, by any substitution whatever, simultaneously altered or simultaneously unaltered, may be called homotypical. Thus all symmetric functions of the same set of quantities are homotypical: $(x+y-z-w)^{2}$ and $x y+z w$ are homotypical, \&c.

It is one of the most beautiful of Lagrange's discoveries in the theory of equations, that, given the value of any function of the roots, the value of any homotypical function may be rationally determined ${ }^{1}$; in other words, that any homotypical function whatever is a rational function of the coefficients of the equation and of the given function of the roots.

The researches of Mr Jerrard are contained in his work, An Essay on the Resolution of Equations, London, Taylor and Francis, 1859. The solution of an equation of the fifth order is made to depend on an equation of the sixth order in $W$; and he conceives that he has shown that one of the roots of this equation is a rational function of another root: "The equation for $W$ will therefore belong to a class of equations of the sixth degree, the resolution of which can, as Abel has shown, be effected by means of equations of the second and third degrees; whence I infer the possibility of solving any proposed equation of the fifth degree by a finite combination of radicals and rational functions."

1 The à priori demonstration shows the cases of failure. Suppose that the roots of a biquadratic equation are $1,3,5,9$; then, given $a+b=8$, we know that either $a=3, b=5$, or else $a=5, b=3$, and in either case $a b=15$; hence in the present case (which represents the general case), $a+b$ being known, the homotypical function $a b$ is rationally determined. But if the roots are $1,3,5,7$ (where $1+7=3+5$ ), then, given $a+b=8$, this is satisfied by $(a, b=3,5)$ or by $(a, b=1,7)$, and the conclusion is $a b=15$ or 7 ; so that here $a b$ is determined, not as before, rationally, but by a quadratic equation. 
The above property of rational expressibility, if true for $W$, will be true for any function homotypical with $W$; and conversely. I proceed to inquire into the form of the function $W$.

The function $W$ is derived from the function $P$, which denotes any one of the quantities $p_{1}, p_{2}, p_{3}$. And if $x_{1}, x_{2}, x_{3}, x_{4}, x_{5}$ are the roots of the given equation of the fifth order, and if $\alpha, \beta, \gamma, \delta, \epsilon$ represent in an undetermined or arbitrary order of succession the five indices $1,2,3,4,5$, and if $\iota$ denote an imaginary fifth root of unity (I conform myself to $\mathrm{Mr}$ Jerrard's notation), then $p_{1}, p_{2}, p_{3}$, and the other auxiliary quantities $t, u$, are obtained from the system of equations:

$$
\begin{aligned}
& x_{a}{ }^{3}+p_{1} x_{a}{ }^{2}+p_{2} x_{a}+p_{3}=t+u, \\
& x_{\beta}{ }^{3}+p_{1} x_{\beta}{ }^{2}+p_{2} x_{\beta}+p_{3}=\imath t+\iota^{4} u, \\
& x_{\gamma}{ }^{3}+p_{1} x_{\gamma}{ }^{2}+p_{2} x_{\gamma}+p_{3}=\iota^{2} t+\iota^{3} u, \\
& x_{\delta}{ }^{3}+p_{1} x_{\delta}{ }^{2}+p_{2} x_{\delta}+p_{3}=\iota^{3} t+\iota^{2} u, \\
& x_{\epsilon}{ }^{3}+p_{1} x_{\epsilon}{ }^{2}+p_{2} x_{\epsilon}+p_{3}=\iota^{4} t+\iota u .
\end{aligned}
$$

If from these equations we seek for the values of $p_{1}, p_{2}, p_{3}, t, u$, we have

$$
1: p_{1}: p_{2}: p_{3}:-t:-u=\Pi_{1}: \Pi_{2}: \Pi_{3}: \Pi_{4}: \Pi_{5}: \Pi_{6},
$$

where $\Pi_{1}, \Pi_{2}, \ldots$ denote the determinants formed out of the matrix

$$
\left|\begin{array}{llllll}
x_{\alpha}{ }^{3}, & x_{a}{ }^{2}, & x_{a}, & 1, & 1, & 1 \\
x_{\beta}{ }^{3}, & x_{\beta}{ }^{2}, & x_{\beta}, & 1, & \iota, & \iota^{4} \\
x_{\gamma}{ }^{3}, & x_{\gamma}{ }^{2}, & x_{\gamma}, & 1, & \iota^{2}, & \iota^{3} \\
x_{\delta}{ }^{3}, & x_{\delta}{ }^{2}, & x_{\delta}, & 1, & \iota^{3}, & \iota^{2} \\
x_{\epsilon}{ }^{3}, & x_{\epsilon}{ }^{2}, & x_{\epsilon}, & 1, & \iota^{4}, & \iota
\end{array}\right|
$$

i.e., denoting the columns of this matrix by $1,2,3,4,5,6$, we have $\Pi_{1}=23456$, $\Pi_{2}=-34561, \Pi_{3}=45612$, \&c. In particular, the value of $\Pi_{1}$ is

$$
=\left|\begin{array}{lllll}
x_{a}^{2}, & x_{a}, & 1, & 1, & 1 \\
x_{\beta}{ }^{2}, & x_{\beta}, & 1, & \iota, & \iota^{4} \\
x_{\gamma}{ }^{2}, & x_{\gamma}, & 1, & \iota^{2}, & \iota^{3} \\
x_{\delta}{ }^{2}, & x_{\delta}, & 1, & \iota^{3}, & \iota^{2} \\
x_{\epsilon}{ }^{2}, & x_{\epsilon}, & 1, & \iota^{4}, & \iota
\end{array}\right|,
$$

and developing, and putting for shortness $\{\alpha \beta\}=x_{\alpha} x_{\beta}\left(x_{\alpha}-x_{\beta}\right)$, \&c., we have

$$
\begin{aligned}
\Pi_{1} & =(\{\alpha \beta\}+\{\beta \gamma\}+\{\gamma \delta\}+\{\delta \epsilon\}+\{\epsilon \alpha\})\left(-2 \iota+\iota^{2}-\iota^{3}+2 \iota^{4}\right) \\
& +(\{\alpha \gamma\}+\{\gamma \epsilon\}+\{\epsilon \beta\}+\{\beta \delta\}+\{\delta \alpha\})\left(+\iota+2 \iota^{2}-2 \iota^{3}-2 \iota^{4}\right)
\end{aligned}
$$


and this is also the form of the other determinants, the only difference being as to the meaning of the symbol $\{\alpha \beta\}$, which, however, in each case denotes a function such that $\{\alpha \beta\}=-\{\beta \alpha\}$. Writing for greater shortness,

$$
\{\alpha \beta \gamma \delta \epsilon\}=\{\alpha \beta\}+\{\beta \gamma\}+\{\gamma \delta\}+\{\delta \epsilon\}+\{\epsilon \alpha\},
$$

and in like manner

$$
\left\{\alpha_{\gamma} \epsilon \delta\right\}=\{\alpha \gamma\}+\{\gamma \epsilon\}+\{\epsilon \beta\}+\{\beta \delta\}+\{\delta \alpha\},
$$

$\Pi_{1}$ is an unsymmetric linear function (without constant term) of $\{\alpha \beta \gamma \delta \epsilon\},\{\alpha \gamma \epsilon \beta \delta\}$; or, what is all that is material, it is an unsymmetric function, containing only odd powers, of $\{\alpha \beta \gamma \delta \epsilon\},\{\alpha \gamma \epsilon \beta \delta\}$.

If for

$$
\alpha \beta \gamma \delta \in
$$

we substitute any one of the five arrangements

$$
\begin{array}{lllll}
\alpha & \beta & \gamma & \delta & \epsilon, \\
\beta & \gamma & \delta & \epsilon & \alpha, \\
\gamma & \delta & \epsilon & \alpha & \beta, \\
\delta & \epsilon & \alpha & \beta & \gamma, \\
\epsilon & \alpha & \beta & \gamma & \delta,
\end{array}
$$

then $\{\alpha \beta \gamma \delta \epsilon\}$ and $\{\alpha \gamma \epsilon \beta \delta\}$ will in each case remain unaltered.

But if we substitute any one of the five arrangements

$$
\begin{array}{lllll}
\alpha & \epsilon & \delta & \gamma & \beta, \\
\epsilon & \delta & \gamma & \beta & \alpha, \\
\delta & \gamma & \beta & \alpha & \epsilon, \\
\gamma & \beta & \alpha & \epsilon & \delta, \\
\beta & \alpha & \epsilon & \delta & \gamma,
\end{array}
$$

then in each case $\{\alpha \beta \gamma \delta \epsilon\}$ and $\left\{\alpha_{\gamma \epsilon} \beta \delta\right\}$ will be changed into $-\{\alpha \beta \gamma \delta \epsilon\}$ and $-\{a \gamma \epsilon \beta \delta\}$ respectively. Hence $\Pi_{1}$ remains unaltered by any one of the first five substitutions; and it is changed into $-\Pi_{1}$ by any one of the second five substitutions. And the like being the case as regards $\Pi_{2}$, \&c., it follows that the quotient $\Pi_{1} \div \Pi_{2}$, or say $P$, remains unaltered by any one of the ten substitutions. Now the 120 permutations of $\alpha, \beta, \gamma, \delta, \epsilon$ can be obtained as follows, viz. by forming the 12 different pentagons which can be formed with $\alpha, \beta, \gamma, \delta, \epsilon$ (treated as five points), and reading each of them off in either direction from any angle. To each of the 12 pentagons there corresponds a distinct value of $P$, but such value is not altered by the different modes of reading off the pentagon; $P$ is consequently a 12 -valued function. 
But there is a more simple form of the analytical expression of such a 12-valued function; in fact, if $[\alpha \beta \gamma \delta \epsilon]$ be any function which is not altered by any one of the above ten substitutions-if, for instance, $[\alpha \beta]$ is a symmetrical function of $x_{\alpha}, x_{\beta}$, and

$$
[\alpha \beta \gamma \delta \epsilon]=[\alpha \beta]+[\beta \gamma]+[\gamma \delta]+[\delta \epsilon]+[\epsilon \alpha],
$$

and therefore

$$
[\alpha \gamma \epsilon \beta \delta]=[\alpha \gamma]+[\gamma \epsilon]+[\epsilon \beta]+[\beta \delta]+[\delta \alpha],
$$

then any unsymmetrical function of $[\alpha \beta \gamma \delta \epsilon]$ and $[\alpha \gamma \epsilon \beta \delta]$ will be a 12 -valued function homotypical with $P$.

$\mathrm{Mr}$ Jerrard's function $W$ is the sum of two values of his function $P$; the substitution by which the second is derived from the first can only be that which interchanges the two functions $[\alpha \beta \gamma \delta \epsilon]$ and $[\alpha \gamma \epsilon \beta \delta]$; and hence any symmetrical function of $[\alpha \beta \gamma \delta \epsilon]$ and $\left[\alpha_{\gamma} \beta \delta \delta\right]$ is a function homotypical with Mr Jerrard's $W$; such symmetric function is in fact a 6 -valued function only. Indeed it is easy to see that the twelve pentagons correspond together in pairs, either pentagon of a pair being derived from the other one by stellation, and the six values of the function in question corresponding to the six pairs of pentagons respectively.

Writing with Mr Cockle and Mr Harley,

$$
\begin{aligned}
& \tau=x_{\alpha} x_{\beta}+x_{\beta} x_{\gamma}+x_{\gamma} x_{\delta}+x_{\delta} x_{\epsilon}+x_{\epsilon} x_{\alpha}, \\
& \tau^{\prime}=x_{a} x_{\gamma}+x_{\gamma} x_{\epsilon}+x_{\epsilon} x_{\beta}+x_{\beta} x_{\delta}+x_{\delta} x_{a},
\end{aligned}
$$

then $\left(\tau+\tau^{\prime}\right.$ is a symmetrical function of all the roots, and it must be excluded; but) $\left(\tau-\tau^{\prime}\right)^{2}$ or $\tau \tau^{\prime}$ are each of them 6-valued functions of the form in question, and either of these functions is linearly connected with the Resolvent Product. In Lagrange's general theory of the solution of equations, if

$$
f \iota=x_{1}+\iota x_{2}+\iota^{2} x_{3}+\iota^{3} x_{4}+\iota^{4} x_{5},
$$

then the coefficients of the equation the roots whereof are $(f i)^{5},\left(f i^{2}\right)^{5},\left(f i^{3}\right)^{5},\left(f i^{4}\right)^{5}$, and in particular the last coefficient $\left(f i i^{2} \imath^{2} i^{3} i^{4}\right)^{5}$, are determined by an equation of the sixth degree; and this last coefficient is a perfect fifth power, and its fifth root, or $f i f i^{2} f i^{3} f i^{4}$, is the function just referred to as the Resolvent Product.

The conclusion from the foregoing remarks is that if the equation for $W$ has the above property of the rational expressibility of its roots, the equation of the sixth order resulting from Lagrange's general theory has the same property.

I take the opportunity of adding a simple remark on cubic equations. The principle which furnishes what in a foregoing foot-note is called the $a$ priori demonstration of Lagrange's theorem is that an equation need never contain extraneous roots; a quantity which has only one value will, if the investigation is properly conducted, be determined in the first instance by a linear equation; one which has two values by a quadratic equation, and so on; there is always enough, and not more than enough, to determine what is required. 
Take Cardan's solution of the cubic equation $x^{3}+q x-r=0$, we have $x=a+b$, and thence $3 a b=-q, a^{3}+b^{3}=r$; and to obtain the solution we write

$$
a^{3} b^{3}=-\frac{q}{27}, \quad a^{3}+b^{3}=r
$$

But these two equations are not enough to precisely determine $x$, they lead to the 9 -valued function

$$
\sqrt[3]{\frac{r}{2}+\sqrt{\frac{r^{2}}{4}+\frac{q^{3}}{27}}}+\sqrt[3]{\frac{r}{2}-\sqrt{\frac{r^{2}}{4}+\frac{q^{3}}{27}}}
$$

in order to precisely determine $x$, it is (as everybody knows) necessary to use the original equation $a b=-\frac{q}{3}$. But seek for the solution as follows; viz. write $x=a b(a+b)$, which gives

$$
3 a^{3} b^{3}=-q, \quad a^{3} b^{3}\left(a^{3}+b^{3}\right)=r
$$

or what is the same thing,

$$
a^{3} b^{3}=-\frac{q}{3}, \quad a^{3}+b^{3}=-\frac{3 r}{q}
$$

these equations give $x=a b(a+b)$, where

$$
a=\sqrt[3]{-\frac{3 r}{2 q}+\sqrt{\frac{9 r^{2}}{4 q^{2}}+\frac{q}{3}}}, \quad b=\sqrt[3]{-\frac{3 r}{2 q}-\sqrt{\frac{9 r^{2}}{4 q^{2}}+\frac{q}{3}}},
$$

which is a 3-valued function only, $a b$ in this case being not given.

2, Stone Buildings, W.C., January 28, 1861. 